\title{
List of Distinguished Reviewers ACM TACO 2014
}

\author{
Manuel Acacio \\ University of Murcia, Spain \\ IBM, USA \\ ETH Zürich, Switzerland \\ University of Crete, Greece \\ UC Riverside, USA \\ North Carolina State University, USA \\ UPC, Spain \\ USC, USA \\ University of Houston, USA \\ Shanghai Jiao Tong University, China \\ University of Pittsburgh, USA \\ University of Texas, Austin, USA \\ University of Pittsburgh, USA \\ Washington University, USA \\ Texas A \& M University, College Station, USA \\ Penn State University, USA \\ Lawrence Livermore National Laboratory, USA \\ Ghent University, Belgium \\ University of Rochester, USA \\ USC, USA \\ UC Irvine, USA \\ University of Texas, Austin, USA \\ Technion, Israel \\ Ghent University, Belgium \\ EPFL, Switzerland \\ Stony Brook University, USA \\ University of Valencia, Spain \\ Carnegie Mellon University, USA \\ University of Edinburgh, UK \\ University of Maryland, USA \\ University of Delaware, USA \\ University of Illinois, Urbana-Champaign, USA \\ Chalmers University of Technology, Sweden \\ Trinity College, Dublin, Ireland \\ Northwestern University, USA \\ National Chiao Tung University, Taiwan \\ EPFL, Switzerland \\ University of Texas, Austin, USA \\ Cambridge University, UK \\ Northwestern University, USA \\ Penn State University, USA \\ University of Minnesota, Twin Cities, USA \\ University of Wisconsin, Madison, USA
}


Manolis Katevenis

Paul Kelly

Omer Khan

Chandra Krintz

Benjamin Lee

Jaejin Lee

Jeng-Kuen Lee

Mikko Lipasti

Xavier Martorell

Gokhan Memik

Maged Michael

Samuel Midkiff

Andreas Moshovos

Trevor Mudge

Dimitrios Nikolopoulos

Dionisios Pnevmatikatos

Valentin Puente

Jose Renau

Saday Sadayappan

Daniel Sanchez

Marco Santambrogio

Yiannakis Sazeides

Resit Sendag

AndréSeznec

Timothy Sherwood

Mary Lou Soffa

Yan Solihin

Daniel Sorin

Ioannis Sourdis

Nigel Topham

Pedro Trancoso

James Tuck

Osman Unsal

Reddi Vijay

Chia-Lin Yang

Pen-Chung Yew

Ayal Zaks

Lixin Zhang
FORTH, Greece

Imperial College, UK

University of Connecticut, USA

UC Santa Barbara, USA

Duke University, USA

Seoul National University, South Korea

National Tsinghua University, Taiwan

University of Wisconsin, Madison, USA

UPC, Spain

Northwestern University, USA

IBM, USA

Purdue University, USA

University of Toronto, Canada

University of Michigan, USA

Queen's University of Belfast, UK

FORTH, Greece

University of Cantabria, Spain

UC Santa Cruz, USA

Ohio State University, USA

MIT, USA

Politecnico di Milano, Italy

University of Cyprus, Cyprus

University of Rhode Island, USA

INRIA, Rennes, France

UC Santa Barbara, USA

University of Virginia, USA

North Carolina State University, USA

Duke University, USA

Chalmers University of Technology, Sweden

University of Edinburgh, UK

University of Cyprus, Cyprus

North Carolina State University, USA

Barcelona Supercomputing Center, Spain

University of Texas, Austin, USA

National Taiwan University, Taiwan

University of Minnesota, USA

Intel, Israel

Institute of Computing Technology, China 